\title{
EDITORIAL
}

\section{Liderazgo, políticas públicas e inclusión en las universidades: Imperativos estratégicos ${ }^{1}$}

A nivel global, la educación universitaria enfrenta requerimientos crecientes. Por una parte, en cuanto debe responder a estándares académicos de calidad, que están siendo cada vez más desafiantes. A la vez, debe abordar las complejas necesidades de estudiantes en desventaja, cuya participación efectiva en el mundo universitario exige políticas de equidad e inclusión ${ }^{2}$.

La inclusión es un concepto polisémico y controversial. En una de las múltiples dimensiones de la inclusión, en el contexto de Chile se ha planteado que, cuando se habla de inclusión en educación superior: "Nos referimos específicamente a la generación de oportunidades de acceso, logro y egreso universitario para todos los jóvenes, especialmente para estudiantes con talento académico que viven en sectores de alta vulnerabilidad social"3.

La incorporación de estudiantes que provienen de contextos de alta vulnerabilidad y marginalización social es un imperativo estratégico en las instituciones universitarias ${ }^{4}$. Las universidades deben ser una expresión de la diversidad de la sociedad, teniendo como requisito esencial de acceso sólo al mérito. Mérito que, por cierto, debe ser debidamente ponderado y definido en atención a las desigualdades de oportunidades que acarrean algunos estudiantes ${ }^{5}$.

No basta con abrir las puertas a los estudiantes de sectores postergados. Esta sola acción no es suficiente, puesto que la verdadera inclusión demanda que los estudiantes en desventaja se mantengan en el sistema y logren sus propósitos académicos, en tiempos razonablemente oportunos, lo cual se torna posible y realizable en la medida que las políticas públicas y las políticas institucionales apoyen efectivamente a los estudiantes marginados ${ }^{6}$.

La evidencia internacional (por ejemplo, ${ }^{7,8,9}$ ) muestra que las políticas públicas juegan un papel fundamental para lograr que la inclusión sea una realidad en las instituciones de educación superior.

Los autores agradecen a la Agencia Nacional de Investigación y Desarrollo de Chile, Proyecto ANID CIE160007.

2 C. Evans, C. Kandiko Howson, A. Forsythe and C. Edwards. "What constitutes high quality higher education pedagogical research?". Assessment \& Evaluation in Higher Education. Vol. $46 \mathrm{~N}^{\circ}$ 4, pp. 525-546. 2021.

3 O. Lizama, F.J. Gil and B. Rahmer. "La experiencia de la inclusión en la educación superior en Chile". Editorial USACH. 2da edición pp. 19. Santiago, Chile. 2018.

4 G. Squires. "Inclusion: Responding to diversity and ideological confusion". Inclusion and Diversity: Communities and Practices Across World. Cambridge Scholars Press. Newcastle, United Kingdom. 2021.

5 F.J. Gil. "Selección universitaria 2013-2014: El ranking de las notas, una decisión impostergable". Mensaje. Vol. 62 № 625, pp. 42-44. 2013.

6 F.J. Gil-Llambías, R. del Valle Martin, M. Villarroel Jorquera and C. Fuentes Vega. "Caracterización y desempeño académico de estudiantes de acceso inclusivo PACE en tres universidades chilenas". Revista Latinoamericana de Educación Inclusiva. Vol. $13 \mathrm{~N}^{\mathrm{o}}$ 2, pp. 259-271. 2019.

7 J.M. Gidley, G.P. Hampson, L. Wheeler and E. Bereded-Samuel. "From access to success: an integrated approach to quality higher education informed by social inclusion theory and practice". Higher Education Policy. Vol. 23 No 1, pp. 123-147. 2010.

8 A. Moriña. "Inclusive education in higher education: Challenges and opportunities". European Journal of Special Needs Education. Vol. $32 \mathrm{~N}^{\circ}$ 1, pp. 3-17. 2017.

9 D. Rodríguez-Gómez, J. Gairín, F. Dovigo, K. Clements, M. Jerónimo, L. Lucas, E. Marin, S. MehtäläL, F.P. Pinheiro, S. Timmis and M. Stîngu. "Access4All: Policies and practices of social development in higher education". Strategies for Facilitating Inclusive Campuses in Higher Education: International Perspectives on Equity and Inclusion. Emerald Publishing Limited, pp. 55-69. Bingley, United Kingdom. 2019. 
Sin embargo, las propias instituciones constituyen un elemento central para lograr la inclusión ${ }^{10}$. Dicho de modo claro, si las autoridades de las instituciones de educación superior asumen a la inclusión como un imperativo estratégico, y como un desafío por el cual vale la pena desplegar esfuerzos relevantes, entonces las posibilidades de las políticas de inclusión éxito serán mayores que en el caso contrario.

Bajo esta mirada, se puede evaluar el rol del liderazgo en las universidades. En efecto, los estilos de liderazgo están definidos por el conjunto de características que poseen quienes ejercen como líderes en una organización ${ }^{11}$. El líder es quien logra movilizar y conducir a otros para que realicen lo que éste desea o requiere en favor de la organización ${ }^{12}$. No todos los líderes tienen las mismas características, ni realizan las mismas actuaciones y, por ende, su desempeño genera diferentes resultados en las instituciones.

Desde el enfoque de la teoría de los escalafones superiores, los directivos toman sus decisiones y adoptan sus actuaciones en función de su interpretación de la realidad, la que está determinada significativamente por sus valores, creencias, y su base cognitiva, que limitan su percepción y campo de visión ${ }^{13}$. Así, diferentes líderes, frente a una misma situación, pueden ver matices distintos de la realidad, tanto en términos de su amplitud como de su profundidad. Por consiguiente, el prisma con el cual los líderes asuman el desafío de la inclusión en las universidades será un determinante estructural del modo cómo la inclusión sea finalmente incorporada y fortalecida al interior de estas instituciones y de la manera cómo se exprese la misma en el quehacer académico y en sus resultados concretos ${ }^{14}$.

Establecido lo anterior, se proponen, sin ánimo exhaustivo, algunos desafíos estratégicos para los líderes en las universidades para promover una inclusión exitosa en la educación universitaria. Primero, los líderes deben desarrollar una conceptualización amplia de la inclusión, que considere todas las diversas formas en que se expresa la marginación, la desigualdad y la falta de oportunidades. Segundo, los líderes deben comprender ellos mismos y transmitir a sus comunidades que la situación de desmedro en que se encuentran algunos grupos de estudiantes no es debida a un déficit de ellos, sino a un sistema social y educacional que posterga a algunos, independientemente de sus talentos. Tercero, los líderes universitarios deben priorizar la inclusión como un objetivo estratégico institucional. Esto es, la inclusión es un propósito central de las universidades, su definición es primordial para éstas, y su logro exitoso es reconocido como un aspecto esencial del desempeño universitario. Cuarto, los líderes en las universidades deben ser capaces de desarrollar estrategias de inclusión en sus organizaciones, que consideren simultáneamente el acceso, la permanencia, la titulación oportuna y la posterior inserción laboral de los estudiantes más vulnerables. Quinto, los líderes universitarios deben comprometer a todo su equipo y a la comunidad académica con los resultados de los procesos formativos de los estudiantes postergados, con su permanencia y titulación en períodos razonablemente oportunos. Sexto, los líderes en las universidades deben instalar las capacidades institucionales en términos físicos, digitales, de procesos y sistemas para fortalecer la formación universitaria y los resultados académicos de todos los estudiantes.

\footnotetext{
${ }^{10}$ A. Taysum. "Case studies of higher education building capacity for education, inclusion, identity and philosophies of trust through the doctorate: The findings". Education Policy as a Roadmap for Achieving the Sustainable Development Goals. Emerald Publishing Limited, pp. 171-196. Bingley, United Kingdom. 2019.

${ }^{11}$ F. Gandolfi and S. Stone. "Leadership, leadership styles, and servant leadership". Journal of Management Research. Vol. 18 No 4, pp. 261-269. 2018.

${ }^{12}$ H. Elrehail, O. L. Emeagwali, A. Alsaad and A. Alzghoul. "The impact of transformational and authentic leadership on innovation in higher education: The contingent role of knowledge sharing”. Telematics and Informatics. Vol. $35 \mathrm{~N}^{\circ} 1$, pp. 55 67. 2018.

13 D.C. Hambrick and P.A. Mason. "Upper echelons: the organization as a reflection of its top managers". Academy of Management Review. Vol. 9 N$^{\circ}$ 2, pp. 193-206. 1984.

14 R.P. Adserias, L.J. Charleston and J.F. Jackson. "What style of leadership is best suited to direct organizational change to fuel institutional diversity in higher education?". Race Ethnicity and Education. Vol. 20 No 3, pp. 315-331. 2017.
} 
Desde luego, las políticas públicas influyen significativamente en la inclusión, pero son insuficientes para generar resultados de largo plazo, que proporcionen una sustentabilidad en un marco de eficacia y eficiencia. En consecuencia, el liderazgo de las autoridades universitarias resulta de la mayor relevancia para impulsar, promover, sostener y ejecutar acciones estratégicas que conduzcan a mayores y necesarios niveles de inclusión.

Liliana Pedraja-Rejas

Facultad de Ingeniería

Universidad de Tarapacá

Arica, Chile

E-mail: 1pedraja@uta.cl
Andrés Bernasconi

Centro Justicia Educacional

Pontificia Universidad Católica de Chile Santiago, Chile

E-mail: abernasconi@uc.cl 